\title{
Preface to the special issue on computing and combinatorics
}

\author{
Yixin $\mathrm{CaO}^{1}(\mathbb{D}) \cdot$ Jianer Chen $^{2}$
}

Published online: 14 February 2019

๑) Springer Science+Business Media, LLC, part of Springer Nature 2019

This special issue of the Journal of Combinatorial Optimization consists of 13 papers, selected from 48 presentations at the 23rd International Computing and Combinatorics Conference (COCOON 2017), taken place in Hong Kong, China, during August 3-5, 2017. COCOON 2017 provided a forum for researchers working in the areas of algorithms, theory of computation, computational complexity, and combinatorics related to computing. The technical program of the conference included 48 contributed papers selected by the Program Committee from 111 full submissions received in response to the call for papers. Sixteen of the articles were invited to this special issue. After the regular peer-review process of the journal, thirteen of them are accepted. One of the invited paper remains in review at this writing; if accepted, it will appear in a later issue of the journal.

We would like to express our gratitude to all reviewers for their hard work and the authors for their contribution. We would also like to thank Editor-in-Chief of the Journal of Combinatorial Optimization, Professor Ding-Zhu Du, for the great support and help in preparing this special issue.

Publisher's Note Springer Nature remains neutral with regard to jurisdictional claims in published maps and institutional affiliations.

$\bowtie$ Yixin Cao

yixin.cao@polyu.edu.hk

Jianer Chen

chen@cse.tamu.edu

1 Department of Computing, Hong Kong Polytechnic University, Hong Kong, China

2 Department of Computer Science and Engineering, Texas A\&M University, College Station, TX 77843 , USA 